\title{
A new test for autonomic cardiovascular and neuroendocrine responses in diabetes mellitus: evidence for early vagal dysfunction
}

Received: 30 June 2004 / Accepted: 23 September 2004 / Published online: 29 December 2004

C) Springer-Verlag 2004

\begin{abstract}
Aims/hypothesis: Diabetic autonomic neuropathy affects many physiological systems, producing a variety of important clinical manifestations. It is associated with high morbidity and mortality, particularly during times of stress. This is thought to be due to an increased risk of cardiac arrhythmias, although the exact mechanisms involved have yet to be fully elucidated. The aim of the present study was to investigate the endocrine, cardiac autonomic and psychological responses of diabetic patients with and without autonomic neuropathy to a single breath of $35 \% \mathrm{CO}_{2}$. Methods: The $35 \% \mathrm{CO}_{2}$ challenge was performed in 20 male diabetic subjects, 11 of whom had autonomic neuropathy. Results: Baseline and stimulated cortisol, prolactin, systolic blood pressure and emotional arousal were similar in the two groups. However, subjects with autonomic neuropathy failed to demonstrate the expected $\mathrm{CO}_{2}$-induced bradycardia seen in the non-neuropathic patients $(p<0.0001)$. Conclusions/ interpretation: The $\mathrm{CO}_{2}$ challenge can be safely and easily administered to produce hypothalamic-pituitary-adrenal axis and cardiac autonomic activation, as well as emotional arousal. The test clearly distinguishes between subjects with and without cardiac autonomic neuropathy and could be an important adjunct to the methods currently available
\end{abstract}

J. M. Kaye · S. L. Lightman

Henry Wellcome Laboratories for Integrative Neuroscience and Endocrinology, University of Bristol,

Bristol, UK

R. J. Corrall

Department of Endocrinology and Diabetes, Bristol Royal

Infirmary,

Bristol, UK

J. M. Kaye $(\bowtie)$

Department of Endocrinology and Diabetes, Sir Charles

Gairdner Hospital,

Verdun Street, Nedlands,

Perth, 6006, WA, Australia

e-mail: joey.kaye@health.wa.gov.au

Tel.: +61-8-93462467

Fax: +61-8-93463221 for the investigation and diagnosis of diabetic autonomic neuropathy.

Keywords Cardiac autonomic neuropathy $\cdot$ Carbon dioxide $\cdot$ Diabetes mellitus $\cdot$ Hypothalamic-pituitaryadrenal axis $\cdot$ Stress

Abbreviations DAN+: with autonomic neuropathy DAN-: without autonomic neuropathy - E:I ratio: expiration : inspiration ratio - HPA: hypothalamicpituitary-adrenal $\cdot$ NA: nucleus ambiguus $\cdot$ SAM: sympatho-adrenomedullary $\cdot \mathrm{VC}$ : vital capacity

\section{Introduction}

Autonomic neuropathy is an important chronic complication of diabetes that produces a variety of clinical features and physiological changes which are related to the widespread involvement of the sympathetic and parasympathetic divisions of the autonomic nervous system $[1,2]$. Damage to peripheral nerves is related to glycaemic control, and the frequency of clinically apparent neuropathy is related to the duration of diabetes and the adequacy of control [1]. The exact pathological mechanism responsible for this is unknown; however, it is likely to be multifactorial in origin and involve both metabolic and vascular factors $[1,3]$.

The prevalence of autonomic neuropathy is highly variable depending on the diagnostic criteria used and the population studied [4], with recent studies in type 1 diabetic patients suggesting prevalence rates of $20-36 \%$ $[4,5]$. Many patients with diabetes demonstrate abnormalities of autonomic function on specific testing without having any clinical symptoms [1]; however, once established, autonomic neuropathy is associated with a significant deterioration in quality of life and increased morbidity and mortality [1, 6-9]. The increased risk of premature death has been demonstrated in several studies [7-9], one of which reported that the 5-year mortality rate is increased by a factor of five in type 1 diabetic patients 
with autonomic neuropathy compared with that in those without neuropathy [7]. The mechanism behind this increased mortality is unclear, although most hypotheses have implicated the increased arrhythmogenic potential of cardiac autonomic neuropathy [8]. However, not all studies have shown an increase in cardiovascular (as opposed to all-cause) mortality, or even an increased prevalence of non-fatal arrhythmias [8]. Furthermore, it has also been noted that the majority of deaths appear to occur during times of acute stress or during sleep [10]. Several studies have therefore examined the integrity of the stress response in people with diabetic autonomic neuropathy. Most of these studies have concentrated on the response to hypoglycaemia, and reduced counter-regulatory hormone responses with impaired sympatho-adrenomedullary (SAM) function are well described $[11,12]$. Responses to other challenges are more variable $[2,13,14]$.

We have been developing a novel test of the human autonomic and neuroendocrine stress response for use in clinical populations. The test, which involves a single breath of $35 \% \mathrm{CO}_{2}$, is safe and easy to administer. It generates a hypothalamic-pituitary-adrenal (HPA) and prolactin response, as well as an autonomic response that has clearly identifiable parasympathetic and sympathetic components [15]. In order to further evaluate the pathogenesis and potential mechanisms that may contribute to the increased mortality experienced by patients with diabetic autonomic neuropathy we performed this test in two groups of diabetic patients, one with autonomic neuropathy and one without.

\section{Subjects and methods}

Subjects Twenty male subjects aged between 18 and 70 years with diabetes of at least 3 years duration were recruited from the Bristol Royal Infirmary. According to the criteria described below, 11 were classified as having autonomic neuropathy (DAN+), whilst the remaining nine had no evidence of autonomic dysfunction (DAN-). Subjects were asked to attend once, where they all received a single breath of $35 \% \mathrm{CO}_{2}$ according to the protocol described below. The Ethics Committee of the United Bristol Healthcare Trust approved the study and all subjects provided written informed consent prior to participation.

Autonomic function studies were performed after a 15-min rest period, but were not analysed until after the completion of the $\mathrm{CO}_{2}$ challenge. On the day of testing, all subjects were asked to attend between 11.00 and 12.00 hours and had their blood glucose level measured on arrival. Testing was only undertaken if subjects had blood glucose levels between 5 and $12 \mathrm{mmol} / \mathrm{l}$. An intravenous line (Venflon; Viggo Spectramed, Helingsborg, Sweden) was placed in an antecubital vein and subjects were asked to rest quietly on a bed prior to autonomic function studies being performed. A 12-lead ECG was recorded and autonomic function studies were performed according to the method of Ewing and Clark [16]. This procedure involved determining the R-R interval from lead II of the
ECG and classifying subjects according to the results of the following tests [13].

1. The supine position test was used to determine the variation in sequential $\mathrm{R}-\mathrm{R}$ intervals after a 1 -min rest (normal $\geq 1.4$, borderline 1.1-1.3, abnormal $\leq 1.0$ ).

2. The expiration/inspiration (E:I) ratio was assessed with the subject breathing at maximal vital capacity (VC) at a regular rate of six breaths per min. During each expiration-inspiration cycle, the ratio of the longest to the shortest $\mathrm{R}-\mathrm{R}$ interval is calculated and the mean of six ratios taken as the E:I ratio (normal $\geq 15 \mathrm{bpm}$, borderline $11-14 \mathrm{bpm}$, abnormal $\leq 10 \mathrm{bpm}$ ).

3. The Valsalva index was calculated by dividing the longest $\mathrm{R}-\mathrm{R}$ interval following a Valsalva manoeuvre by the shortest $\mathrm{R}-\mathrm{R}$ interval during the manoeuvre (normal $\geq 1.21$, abnormal $\leq 1.20$ ).

4. Postural hypotension was determined by comparing the supine systolic BP after a 5-min rest with the systolic BP after $1 \mathrm{~min}$ in the standing position (normal $<20 \mathrm{mmHg}$, abnormal $>20 \mathrm{mmHg}$ ).

Using these criteria, subjects were classified as having no autonomic neuropathy (all four tests normal; DAN-) or as having definite autonomic neuropathy (first three tests all abnormal; DAN+) with or without postural hypotension (depending on the result of the fourth test).

Procedure The $\mathrm{CO}_{2}$ test involves taking a single VC breath of a mixture of $35 \% \mathrm{CO}_{2}, 65 \% \mathrm{O}_{2}$ and has been described in detail elsewhere [15]. Briefly, the device used for administering the gas comprises a 10-1 silicone 'Douglas' bag (Hans Rudolph, Kansas City, MO, USA) filled with the gas mixture which is obtained from a commercial source (BOC Gases, Guildford UK). The Douglas bag is connected via an analogue flow meter (Ohmeda Medical, Columbia, MD, USA) to a manual three-way valve (Hans Rudolph). One of the remaining two ports of the three-way valve is open to air, and the other is attached to a silicone mouthpiece (3-cm diameter). Subjects have their nose occluded with a nose-clip whilst they breathe through the mouthpiece. The three-way valve allows the operator to control whether the subject is breathing room air or the gas mixture. Inspired VC is determined using the flow meter.

After completing the autonomic function testing, subjects were shown the device used for the delivery of the $\mathrm{CO}_{2}$ breath and instructed in its use. All subjects received the same instructions. Subjects were told that they would have their nose occluded with a nose-clip whilst they breathed room air through the mouthpiece. When instructed, they would be asked to take a deep breath in and exhale fully. The operator would then turn the tap to open the port of the Douglas bag, and the subject would be asked to take one full deep inspiration and hold it for a slow count of four. The operator would subsequently turn the tap back to the original position, after which the subjects would exhale and then breathe normally. With the Douglas bag disconnected, subjects performed a number of practice breaths in order to get used to using the device, 
understand the instructions, and to record a baseline $\mathrm{VC}$ breath. As has been demonstrated previously, activation of neuroendocrine systems requires a full $\mathrm{VC}$ breath to be taken. The test breath VC was measured and compared with a baseline VC air breath. Exposure was considered adequate only if the test breath was at least $80 \%$ of the baseline breath.

Subjects were excluded if they had poorly controlled diabetes (defined as a recent $\mathrm{HbA}_{1} \mathrm{c} \geq 10 \%$ ), uncontrolled hypertension, a history of recent angina or ischaemic heart disease, or any history of cerebrovascular disease. Other exclusion criteria included age $>70$ years, a history of asthma, or a history of panic disorder or severe anxiety. Patients were allowed to remain on their usual medications provided there had been no recent (within 8 weeks) change and they were not taking exogenous glucocorticoids, antidepressants, antipsychotics, anti-anxiety agents, $\beta 2$ agonists or centrally acting antihypertensives. Other antihypertensives were allowed, and one subject (in the autonomic neuropathy group) was taking a $\beta$-blocker. Subjects were asked to avoid consuming cigarettes, alcohol and caffeine for $24 \mathrm{~h}$ prior to testing.

Monitoring Cardiovascular monitoring commenced $5 \mathrm{~min}$ before the test breath and continued for 5 min after. Systolic BP and heart rate were recorded automatically every minute using an automated Dynamap cardiovascular monitor (Critikon, Tampa, FL, USA).

Subjective feelings of anxiety, fear and breathlessness were recorded using standard $100-\mathrm{mm}$ visual analogue scales. A similar analogue scale was employed to record somatic symptoms of panic using a 39-point questionnaire [17]. Questionnaires were administered immediately before and immediately after the $\mathrm{CO}_{2}$ exposure.

Two baseline blood samples were taken at -10 and 0 min before exposure, and three post-exposure samples were taken at 10, 20 and $30 \mathrm{~min}$. On each occasion, $5 \mathrm{ml}$ of venous blood was collected and assayed within $24 \mathrm{~h}$ for cortisol and prolactin using standard immunometric assays (Immulite 2000; DPC, Los Angeles, CA, USA). The intraand inter-assay CVs were 5.3 and $7.2 \%$, respectively, for cortisol and 2.5 and $6.9 \%$, respectively, for prolactin.

Statistical analysis Data are presented as means \pm SEM. Two-way repeated measures ANOVA was used to determine between-group differences and time effects, and two-tailed $t$-tests were used to compare single time-point data including baseline differences. Fisher's exact test was used to compare categorical outcomes. A $p$ value of less than 0.05 was considered statistically significant.

\section{Results}

The $\mathrm{CO}_{2}$ challenge was well tolerated by all participants, with no significant adverse effects recorded. Anxiety symptoms were all mild and transient, lasting from a few seconds to a few minutes only. One individual (from the DAN- group) did not take an adequate breath of $\mathrm{CO}_{2}(\mathrm{VC}$ $<80 \%$ of baseline), and data for this individual was excluded from the analysis.
Table 1 Baseline characteristics of diabetic subjects with and without autonomic neuropathy

Values shown are means \pm SEM or $n(\%)$, unless stated otherwise

\begin{tabular}{|c|c|c|c|}
\hline & $\begin{array}{l}\text { Control group } \\
(n=8)\end{array}$ & $\begin{array}{l}\text { Autonomic neuropathy group } \\
(n=11)\end{array}$ & $p$ value \\
\hline Age (years) & $40.6 \pm 5.3$ & $60.0 \pm 2.4$ & 0.002 \\
\hline Age range & $24-36$ & $40-69$ & - \\
\hline \multicolumn{4}{|l|}{ Diabetes type } \\
\hline Type 1 & $6(75)$ & $6(55)$ & NS \\
\hline Type 2 & $2(25)$ & $5(45)$ & NS \\
\hline Duration of diabetes (years) & $12.4 \pm 3.6$ & $25.9 \pm 4.0$ & 0.03 \\
\hline Range of duration & $3-36$ & $3-43$ & - \\
\hline \multicolumn{4}{|l|}{ Complications } \\
\hline Diabetic retinopathy & $3(38)$ & $7(63)$ & NS \\
\hline $\begin{array}{l}\text { Nephropathy (including } \\
\text { microalbuminuria) }\end{array}$ & $0(0)$ & $5(45)$ & 0.04 \\
\hline Peripheral neuropathy & $2(25)$ & $10(91)$ & 0.006 \\
\hline \multicolumn{4}{|l|}{ Cardiovascular parameters } \\
\hline Resting blood pressure (mmHg) & $137 / 70$ & $135 / 74$ & NS \\
\hline Postural hypotension & - & $3(27)$ & - \\
\hline Resting heart rate (bpm) & $69.3 \pm 3.2$ & $76.4 \pm 3.5$ & NS \\
\hline \multicolumn{4}{|l|}{ Antihypertensive medications } \\
\hline Nil & $7(87.5)$ & $5(45)$ & NS \\
\hline ACE inhibitor alone & $1(12.5)$ & $2(18)$ & - \\
\hline ACE inhibitor plus other & - & $3(27)$ & - \\
\hline Angiotensin II receptor antagonist & - & $1(9)$ & - \\
\hline$\beta$-Blocker & - & $1(9)$ & - \\
\hline
\end{tabular}


Fig. 1 Cortisol and prolactin responses to $35 \% \mathrm{CO}_{2}$ in diabetic subjects with and without autonomic neuropathy. Baseline concentrations of cortisol (a) and prolactin (b) are shown along with the maximum stimulated cortisol (c) and prolactin $(\mathbf{d})$ responses in 11 subjects with autonomic neuropathy $(\mathrm{DAN}+)$ and eight subjects without (control). The data shown are means \pm SEM a
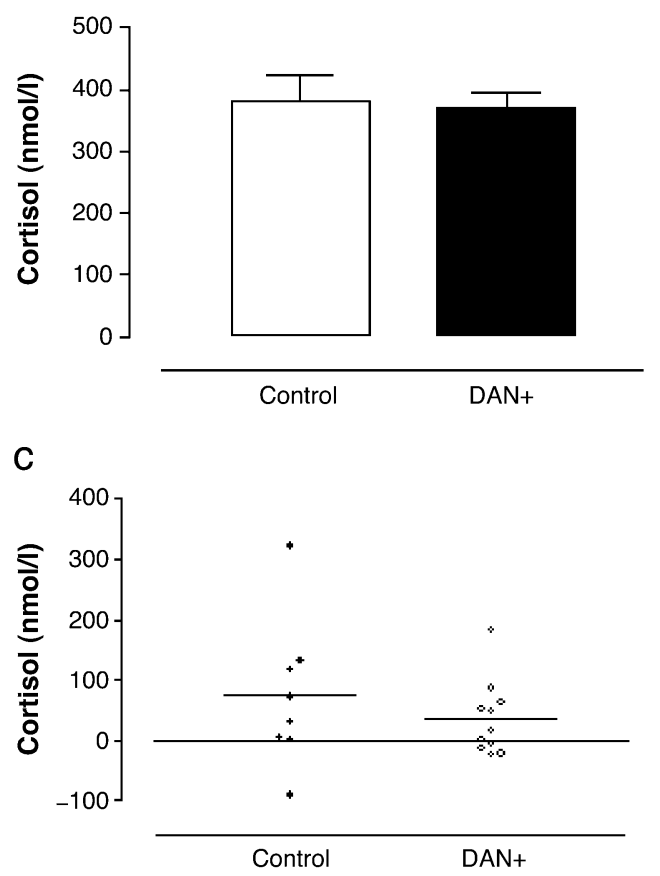

b

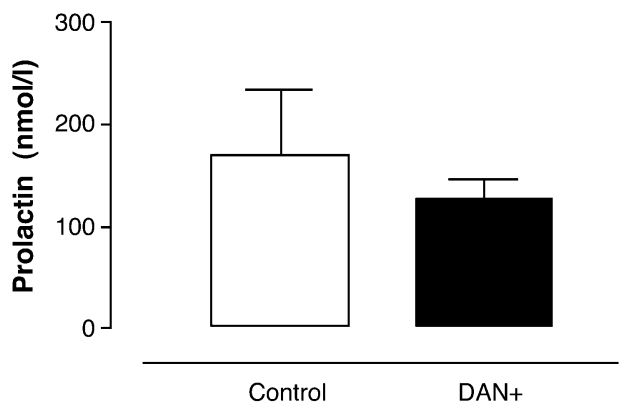

d

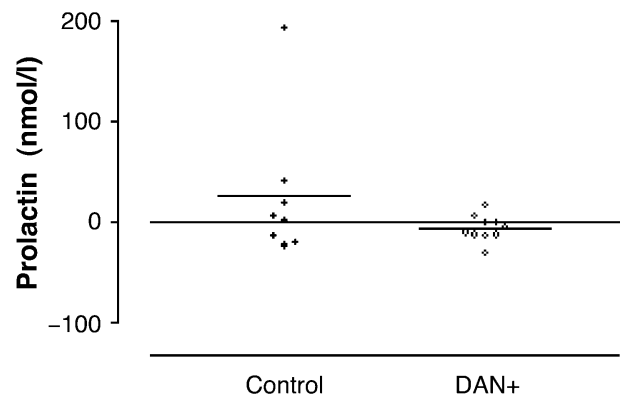

Compared with those in the DAN- group, subjects in the DAN+ group were significantly older $(60.0 \pm 2.4$ vs $40.6 \pm 5.23$ years, $p=0.002)$ and had a longer duration of diabetes $(25.9 \pm 4.0$ vs $12.4 \pm 3.6$ years, $p=0.03)$ (Table 1$)$. In addition, subjects in the DAN+ group were more likely to have other microvascular complications of diabetes, and were more likely to be on antihypertensive medications. The proportion of subjects with type 1 diabetes was not significantly different between the two groups. Three (27\%) of the $11 \mathrm{DAN}+$ subjects also had postural hypotension.

The two groups had similar cortisol and prolactin levels at baseline (Fig. 1a, b). Cortisol levels increased significantly in both groups in response to a single breath of $35 \% \mathrm{CO}_{2}(p=0.02)$; the maximum change in cortisol levels was similar for the two groups (Fig. 1c). The prolactin response to $35 \% \mathrm{CO}_{2}$ did not reach statistical significance in either group (Fig. 1d).
Fig. 2 Cardiovascular responses to $35 \% \mathrm{CO}_{2}$ in diabetic subjects with and without autonomic neuropathy. The systolic $\mathrm{BP}$ response is shown over time (a) with the peak response (b). Similarly, heart rate responses are shown over time (c) with the peak response (d). The data shown are the means \pm SEM for 11 subjects with autonomic neuropathy $(\mathrm{DAN}+)$ and eight without (control). ${ }^{*} p<0.0001$ vs control
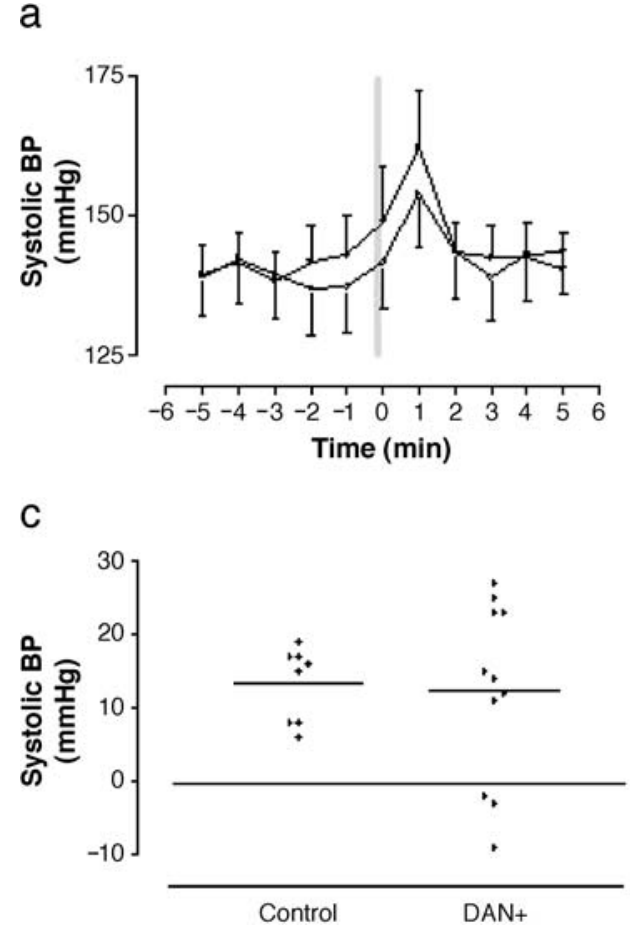
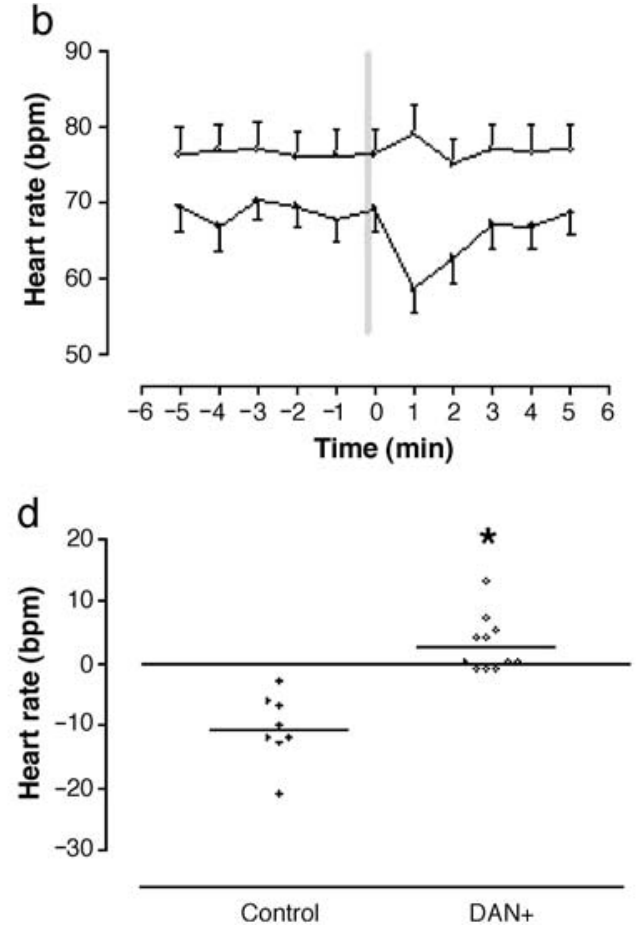


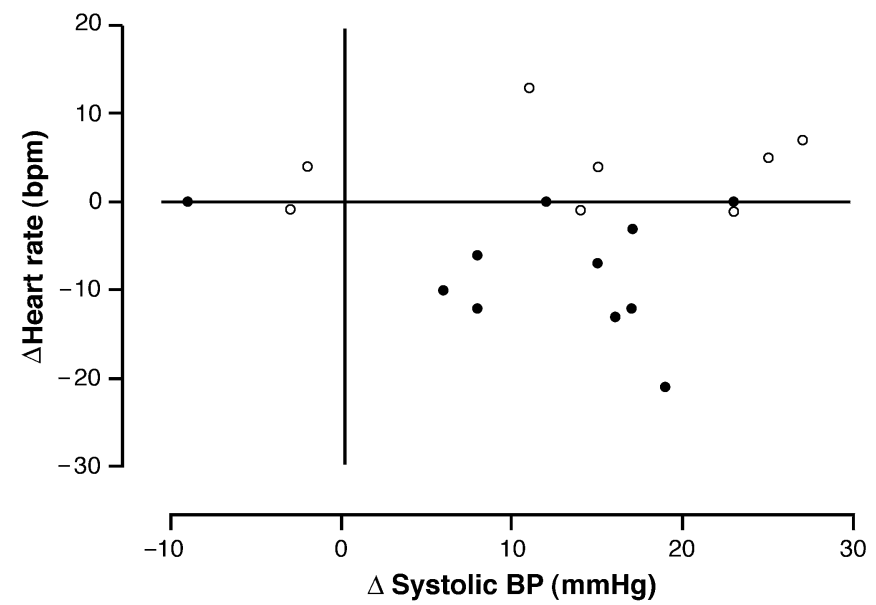

Fig. 3 Relationship between the maximum change in heart rate and the maximum change in systolic BP following a single breath of $35 \% \mathrm{CO}_{2}$ in 11 subjects with autonomic neuropathy $(\circ)$ and eight without $(\bullet)$

a
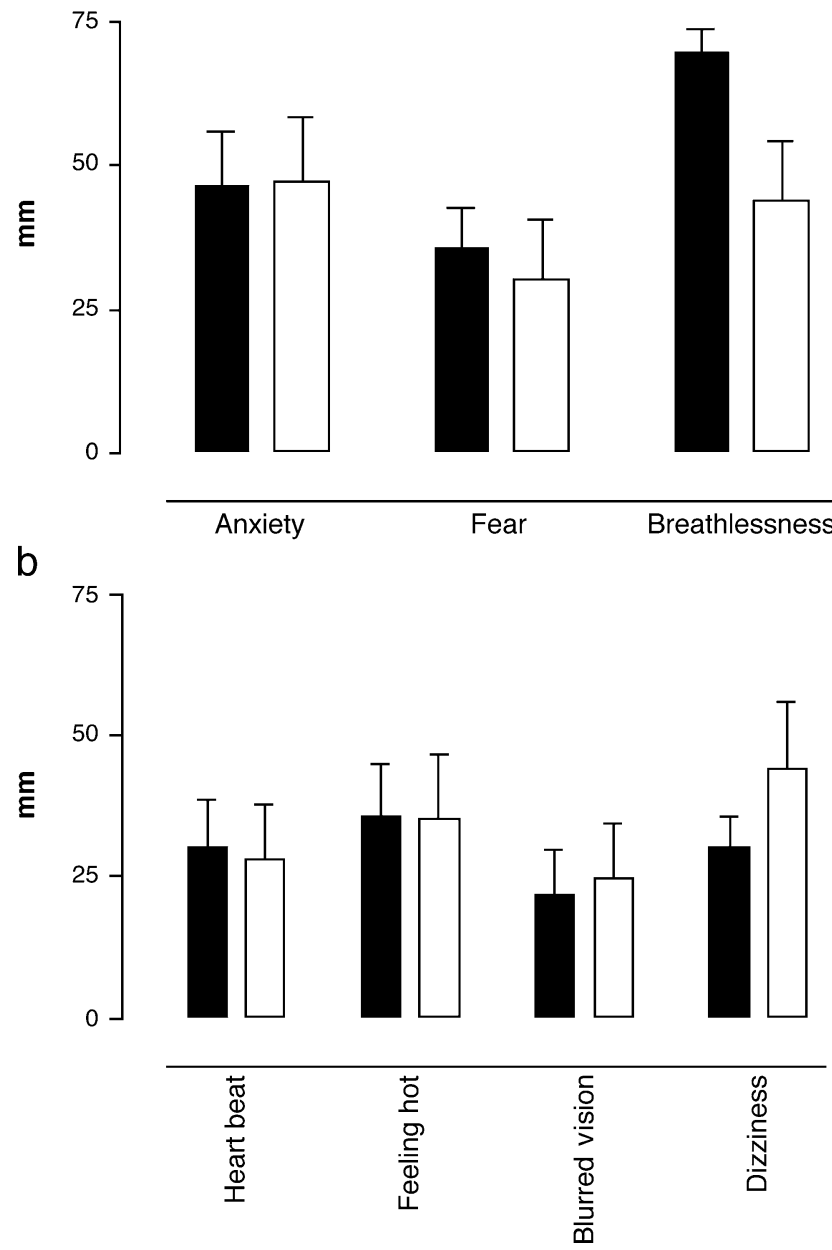

Fig. 4 Psychological responses to $35 \% \mathrm{CO}_{2}$ in diabetic subjects with and without autonomic neuropathy. The changes in subjective anxiety, fear and breathlessness immediately following the challenge are shown (a), along with the changes in some commonly experienced somatic sensations (b) in 11 subjects with autonomic neuropathy (white bars) and eight without (black bars). The data shown are means \pm SEM
As shown in Table 1 and Fig. 2a, the DAN- and DAN+ groups had similar blood pressures at baseline (135/74 and $137 / 70 \mathrm{mmHg}$, respectively). Both groups showed a significant pressor response to $35 \% \mathrm{CO}_{2}(p=0.03)$; the change in blood pressure from baseline was not significantly different between the DAN - and DAN + groups $(+13.2 \pm 1.7$ vs $+12.3 \pm 3.7 \mathrm{mmHg}$, respectively) (Fig. 2b). At baseline, heart rates were consistently higher in the DAN+ group than in the DAN- group (76 \pm 3.5 vs $69 \pm 3.2 \mathrm{bpm})$ (Fig. 2c); however, the between-group difference did not reach statistical significance $(p=0.17)$. There was a significant difference between the two groups in terms of the heart rate response to $35 \% \mathrm{CO}_{2}(p<0.0001 ; F=44.4)$. Subjects in the DAN- group demonstrated a significant bradycardic response that was similar to that seen in normal healthy volunteers $(-10.5 \pm 1.9 \mathrm{bpm}, p=0.001$ compared with baseline). Conversely, none of the DAN+ group demonstrated a bradycardic response; the mean maximum change in heart rate in this group was $+2.7 \pm 1.3 \mathrm{bpm}$ ( $p=0.09$ compared with baseline) (Fig. 2d).

No correlation was observed between the maximum change in heart rate and the maximum change in systolic BP in either group (Fig. 3).

At baseline, anxiety, fear and somatic symptoms of anxiety were minimal in all subjects and did not differ between the two groups. In response to $35 \% \mathrm{CO}_{2}$ there were significant increases in subjective feelings of anxiety, fear, breathlessness and in some specific somatic sensations of fear $(p<0.001)$. The maximum change in these feelings was not significantly different between the two groups (Fig. 4a, b).

\section{Discussion}

In this study we have evaluated the effects of diabetic autonomic neuropathy on the neuroendocrine, autonomic and psychological responses to a novel $35 \% \mathrm{CO}_{2}$ stress paradigm. Despite baseline differences in the age of subjects, duration of diabetes and the presence of microvascular complications between the two groups, there were no significant differences in resting hormonal, cardiovascular or psychological parameters. Similarly, the presence of autonomic neuropathy did not impair the cortisol, blood pressure or psychological responses to $\mathrm{CO}_{2}$. There was, however, a striking difference between the two groups with respect to the heart rate response. The expected response, as determined from previous studies of the $\mathrm{CO}_{2}$ challenge in healthy volunteers, includes initial bradycardia followed by a noradrenaline-mediated increase in total peripheral resistance and a pressor response [15, 18]. Although all of the DAN- subjects had a bradycardic response, this was not seen in any of the DAN+ patients.

The bradycardia appears to be regulated independently of the pressor response and most likely involves the direct activation of brainstem vagal nuclei by $\mathrm{CO}_{2}$. This is supported by the lack of any correlation between the change in heart rate and the pressor response in either group, indicating that the bradycardia is not simply a reflex baro- 
receptor response. Cardiac autonomic neuropathy is typically diagnosed using a battery of manoeuvres designed to evaluate predominantly efferent cardiac vagal activity [19] by assessing $\mathrm{R}-\mathrm{R}$ interval variability and baroreceptor reflexes. In a review of these various manoeuvres it was apparent that, in diabetic autonomic neuropathy, parasympathetically mediated heart rate control is affected earlier than sympathetically mediated vasomotor control. Furthermore, responses to one test of vagal activity may differ from responses to another test $[19,20]$. By examining responses to direct vagal stimulation, this test may offer some additional insights into the potential mechanism responsible for the increased mortality observed in patients with cardiac autonomic neuropathy. The Polyvagal Theory [21] distinguishes between the rhythmic beat-to-beat variations in heart rate [mediated by phylogenetically newer myelinated fibres from the nucleus ambiguus (NA)] and the marked bradycardia (mediated by more primitive unmyelinated fibres from the dorsal motor nucleus) that is occasionally observed during acute stress. It has been postulated that withdrawal of the NA component of the vagal response during acute stress allows sympathetic stimulation to predominate. However, the removal of this component leaves the individual susceptible to the effects of excessive neurogenic bradycardia if sympathetic compensation is inadequate, and this is the likely explanation for neurogenic syncope $[21,22]$. In addition, this is probably the mechanism responsible for the bradycardia seen in normal volunteers in response to the $35 \% \mathrm{CO}_{2}$ challenge. It seems likely that the parasympathetic neuropathy associated with diabetes mellitus affects the outflow from both of the vagal nuclei that would normally act to protect the individual from excessive sympathetic stimulation, which may then increase the likelihood of cardiac tachyarrhythmias $[8,21,22]$.

Baseline and stimulated cortisol responses were equivalent in subjects with and without diabetic autonomic neuropathy. This suggests that the HPA axis response to this challenge remains intact in diabetic subjects and is independent of peripheral autonomic dysfunction. However, it should be noted that subjects in the present study were matched in terms of glycaemic control and all had relatively normal glucose levels at the time of testing, and previous studies have reported that uncontrolled diabetes and acute hyperglycaemia or hypoglycaemia may be associated with altered HPA axis activity $[12,23]$. Cortisol responses to graded exercise have been shown to be impaired in diabetic subjects with autonomic neuropathy, albeit only at maximum workload [14, 24], and it has been suggested that this is due to an impairment in sympathetic afferent impulses [24]. Exercise at maximal workload is a complex stressor that involves other neurohormonal pathways, such as growth hormone (which is also blunted in those with diabetic autonomic neuropathy), and the HPA axis response to exercise is almost certainly mediated through different pathways to those involved in the response to $\mathrm{CO}_{2}$ challenge [14].

Reduced circulating and stimulated levels of prolactin have previously been demonstrated in patients with poorly controlled diabetes and in laboratory animals with induced diabetes $[25,26]$. The mechanism responsible for this is not fully understood; however, recent evidence suggests that a reduction in lactotroph number within the pituitary is involved [26]. This may explain the lack of any significant prolactin response to $\mathrm{CO}_{2}$ in both groups compared with that previously observed in non-diabetic healthy volunteers, who typically demonstrate significant prolactin release in response to this challenge [15].

Finally, emotional arousal in these two groups of patients, as measured by subjective anxiety, fear, breathlessness and somatic symptoms of fear, was no different to that seen in healthy volunteers, with autonomic neuropathy having no additional effect on anxiety or other psychological responses. This would appear to reinforce the notion that psychological responses to $35 \% \mathrm{CO}_{2}$ appear to be mediated through pathways involving central chemoreceptors which are probably stimulated independently but may subsequently influence hypothalamic (HPA and prolactin) and brainstem (SAM) centres through reciprocal connections.

In conclusion, the present study demonstrates normal cortisol and psychological responses to a novel stressor in subjects with and without diabetic autonomic neuropathy, but also indicates that these two groups show significantly different cardiovascular responses. The failure of subjects with autonomic neuropathy to demonstrate a bradycardic response to the $\mathrm{CO}_{2}$ test, despite an equivalent pressor response, suggests a differential sensitivity of the parasympathetic and sympathetic nervous systems to autonomic neuropathy and indicates a potential pathway through which cardiac tachyarrhythmias may occur. It should be noted that $\mathrm{R}-\mathrm{R}$ variability declines with age, and some of the differences observed in this study may be related to the significant difference in age between the two groups examined. Finally, the further development of this test as a simple and safe means of investigating functional impairments of the sympathetic and parasympathetic nervous system may provide a useful screening tool for the investigation and diagnosis of diabetic autonomic neuropathy.

Acknowledgements This was work supported by an F. A. Hadley Travelling Fellowship from the University of Western Australia (Crawley, WA, Australia), the Neuroendocrinology Charitable Trust, UK and The Charitable Trustees of the United Bristol Healthcare Trust. There are no conflicts of interest associated with this work.

\section{References}

1. Ward JW (1992) Diabetic neuropathy. In: Alberti KGMM, Defronzo RA, Keen H, Zimmet P (eds) International textbook of diabetes mellitus. Wiley, Chichester, pp 1385-1414

2. Forst T, Pfutzner A, Jahn C et al (1996) Decreased sympathoadrenal activity in diabetic patients with autonomic dysfunction following mental stress. J Auton Nerv Syst 61:31-36

3. Simmons Z, Feldman EL (2002) Update on diabetic neuropathy. Curr Opin Neurol 15:595-603 
4. Kempler P, Tesfaye S, Chaturvedi N et al (2002) Autonomic neuropathy is associated with increased cardiovascular risk factors: the EURODIAB IDDM Complications Study. Diabet Med 19:900-909

5. Stella P, Ellis D, Maser RE, Orchard TJ (2000) Cardiovascular autonomic neuropathy (expiration and inspiration ratio) in type 1 diabetes. Incidence and predictors. J Diabetes Its Complicat $14: 1-6$

6. Ziegler D (2001) Diagnosis and treatment of diabetic autonomic neuropathy. Curr Diabetes Rep 1:216-227

7. O'Brien IA, McFadden JP, Corrall RJ (1991) The influence of autonomic neuropathy on mortality in insulin-dependent diabetes. Q J Med 79:495-502

8. Wheeler SG, Ahroni JH, Boyko EJ (2002) Prospective study of autonomic neuropathy as a predictor of mortality in patients with diabetes. Diabetes Res Clin Pract 58:131-138

9. Ziegler D (2002) Diabetic autonomic neuropathy. Cardiac sympathetic "dysinnervation," QT interval prolongation, and mortality. Clinic Auton Res 12:349-352

10. Tantucci C, Bottini P, Fiorani C et al (2001) Cerebrovascular reactivity and hypercapnic respiratory drive in diabetic autonomic neuropathy. J Appl Physiol 90:889-896

11. Meyer C, Grossmann R, Mitrakou A et al (1998) Effects of autonomic neuropathy on counterregulation and awareness of hypoglycemia in type 1 diabetic patients. Diabetes Care 21: 1960-1966

12. Chan O, Chan S, Inouye K, Shum K, Matthews SG, Vranic M (2002) Diabetes impairs hypothalamo-pituitary-adrenal (HPA) responses to hypoglycemia, and insulin treatment normalizes HPA but not epinephrine responses. Diabetes 51:1681-1689

13. Granados G, Garay-Sevilla ME, Malacara JM, Wrobel-Zasada K, Rivera-Cisneros A (2000) Plasma epinephrine and norepinephrine response to stimuli in autonomic neuropathy of type 2 diabetes mellitus. Acta Diabetol 37:55-60

14. Hilsted J, Galbo H, Christensen NJ (1980) Impaired responses of catecholamines, growth hormone, and cortisol to graded exercise in diabetic autonomic neuropathy. Diabetes 29:257262
15. Kaye JM, Buchanan F, Kendrick A et al (2004) Acute carbon dioxide exposure in healthy adults: evaluation of a novel means of investigating the stress response. J Neuroendocrinol 16:256264

16. Ewing DJ, Clark BF (1982) Diagnosis and management of diabetic autonomic neuropathy. BMJ 285:916

17. Nutt DJ, Glue P, Lawson C, Wilson S (1990) Flumazenil provocation of panic attacks. Arch Gen Psychiatry 47:917-925

18. Bailey J, Argyropoulos SV, Lightman SL, Nutt DJ (2003) Does the brain noradrenaline network mediate the effects of the $\mathrm{CO}_{2}$ challenge? J Psychopharmacol 17:252-259

19. Bennett T, Farquhar IK, Hosking DJ, Hampton JR (1978) Assessment of methods for estimating autonomic nervous control of the heart in patients with diabetes mellitus. Diabetes 27:1167-1174

20. Bennett T, Hosking DJ, Hampton JR (1980) Cardiovascular responses to graded reductions of central blood volume in normal subjects and in patients with diabetes mellitus. Clin Sci 58:193-200

21. Porges SW (1995) Orienting in a defensive world: mammalian modifications of our evolutionary heritage. A polyvagal theory. Psychophysiology 32:301-318

22. Reed SF, Ohel G, David R, Porges SW (1999) A neural explanation of fetal heart rate patterns: a test of the Polyvagal Theory. Dev Psychobiol 35:108-118

23. Williams JG, Morris AI, Hayter RC, Ogilvie CM (1984) Respiratory responses of diabetics to hypoxia, hypercapnia, and exercise. Thorax 39:529-534

24. Chan O, Inouye K, Riddell MC, Vranic M, Matthews SG (2003) Diabetes and the hypothalamo-pituitary-adrenal (HPA) axis. Minerva Endocrinol 28:87-102

25. Iranmanesh A, Veldhuis JD, Carlsen EC et al (1990) Attenuated pulsatile release of prolactin in men with insulin-dependent diabetes mellitus. J Clin Endocrinol Metab 71:73-78

26. Arroba AI, Frago LM, Paneda C, Argente J, Chowen JA (2003) The number of lactotrophs is reduced in the anterior pituitary of streptozotocin-induced diabetic rats. Diabetologia 46:634-638 